\title{
Balancing Caregiver and Clinician Needs in a Mobile Health Informatics Tool for Preterm Infants
}

\author{
Karen P. Tang, Sen H. Hirano, Karen G. Cheng, Gillian R. Hayes \\ Donald Bren School of Information and Computer Sciences \\ University of California, Irvine \\ \{kptang, shirano, gillianrh\}@ics.uci.edu, kgcheng@uci.edu
}

\begin{abstract}
Preterm infants have significantly higher rates of functional limitations and are at risk for delays in cognitive, motor, and other skills. Through high-risk infant follow-up (HRIF) programs and other early detection methods, these delays and complications can be reduced. However, these interventions require substantial resources and can create extra burden on families. In this paper, we present the results of a qualitative design study to understand the needs of these families and their professional caregivers. These results reveal diverse challenges facing parents and clinicians attempting to document, understand, and share infant health data that must be balanced in any pervasive health solution. In particular, parents described struggling with when to collect data, while clinicians reported being more concerned about what and how parents track infant health. Based on these results, we present five design implications for applications for preterm infant care, which we incorporated into the design of Estrellita, a mobile health informatics tool to support caregivers of preterm infants.
\end{abstract}

Keywords-preterm infants, prematurity, health informatics, capture and access, clinicians, pediatric informatics, mobile devices

\section{INTRODUCTION}

More than $12 \%$ of all US births each year are preterm, i.e. delivered prior to 37 weeks gestation [1]. Most of these infants have low birth weight (LBW) and significantly higher rates of functional limitations or developmental problems when compared with those with normal birth weight (NBW) [2, 3]. In particular, preterm infants are at risk for delays in cognitive, language, motor, and sensory processing skills [3, 4]. To mitigate these challenges, parents must adopt a long-term management plan to care for initial medical complications as well as ongoing developmental concerns. Furthermore, during the transition from the hospital to home, substantial challenges await parents, including the burden and stress of caring for a preterm infant, the additional effort of documenting the infant's progress, and the confusion and difficulties in communicating with clinicians, friends, and family about their child [5].

Past work in neonatal care has shown that regular monitoring can provide early identification of developmental delay. Additionally, early intervention and rehabilitation can minimize the long-term effects of developmental delay [3, 4, 6]. Typically, such monitoring is done by state-funded highrisk infant follow-up (HRIF) programs, through which healthcare professionals see preterm infants about every six months. Between appointments, however, clinicians have limited visibility into the health of the infants. Thus, in this work, we focused on using mobile technologies to augment existing HRIF programs and enable data sharing in near real- time among interested stakeholders. Gathering and responding to health data more quickly can help reduce parental stress and ultimately improve health outcomes for the child [7].

Although HRIF programs support families in getting additional resources for early intervention, they are costly to run. In this work, we are interested in how pervasive computing tools can reduce the expensive human resources required for HRIF work. Recent advances in smartphones enable these tools to be integrated into the caregiver experience at a low cost. Using these mobile devices as health informatics tools can in turn lead to early identification of potential developmental problems in a cost-effective and efficient manner.

In this paper, we describe the results of an interview study and extensive design process that led to the development of a mobile health application called Estrellita, a tool to support caregivers of preterm infants. Estrellita addresses the common burdens of tracking data and balances the sometimescompeting needs of parents, clinicians, and other professionals. We present the diverse challenges that parents and clinicians face when attempting to document, understand, and share infant health data. Based on these findings, we outline the design implications that are broadly applicable to applications for preterm infant care and demonstrate how these are built into our prototype system, Estrellita. We conclude by discussing design challenges we encountered in our process that can be broadly applicable to other pervasive health applications.

\section{RELATED WORK}

Children possess unique physiology, come from a wide range of backgrounds, and experience diseases that are largely unique to them [8]. These factors make them particularly unique and complex patients, with complex health information needs. Yet despite calls for pediatric-specific health tools [8], little has been published focusing on pediatric informatics, and even less on the specific needs of preterm infants. In one recent project relating to preterm infants, Gray et al. evaluated an Internet-based telemedicine program called Baby CareLink, which allowed parents to remotely check on their infant during his stay in the hospital's Neonatal Intensive Care Unit (NICU) [9]. Although these types of tools are important for inpatient care, they neglect the time after discharge. Motorola is doing work related to transitioning from the NICU to the home [10]; however, the focus of Estrellita is to support in-home care.

Past work has shown that even parents of healthy children struggle with data tracking tasks [11] and that families coping with children with chronic illnesses may struggle even more [5, 12]. Thus, researchers have begun to investigate the design of 
systems to support physiological [11] and developmental [13] health documentation in families. Using capture and access technologies [14], data can be automatically, semiautomatically, or manually recorded and made available for later review. Through the deployment of one such system, BabySteps, Kientz et al. found that parents were willing and able to record information about their children's progress [13]. However, they required additional motivation to do so in the form of personal artifacts, such as creating photo albums.

Several commercial products exist to help parents record data about their infants. The majority of these commercial applications (e.g. Trixie Tracker [15]) typically focus on helping parents to track health information like an infant's feeding times, diaper changes, and sleep schedules. However, these systems tend to favor an overly flexible design whereby parents can track almost anything. In our work, we discovered that some parents found this approach burdensome and overwhelming, particularly when caring for preterm infants.

This work builds on the existing literature to offer new contributions. In particular, the results of our formative study presented here indicates the need to balance tensions among the various stakeholders and to use a holistic approach to wellness tracking that considers both maternal and infant health data.

\section{METHODS}

Our approach for designing an effective mobile health informatics tool is to use qualitative methods, including interviews and observations $[16,17]$, to better understand the data capture and access needs for caregivers of preterm infants. Following these interviews, we conducted participatory design exercises [18] over 18 months to develop the Estrellita system.

\section{A. Phase 1: Interviews with Parents and Professionals}

In the first phase of our user-centered design process, our research team conducted interviews with 29 participants living in Southern California between November 2009 and June 2010. These individuals included 18 caregivers (17 mothers, 1 aunt), who were of medium to low socioeconomic status, and 11 healthcare professionals. For simplicity of reading, we refer to familial caregivers as parents or families in the remainder of this paper. In our sample, in nearly every case, the mother declared herself as the primary caregiver responsible for documenting her infant's health and communicating this information to others. At the time of our study, all parents were caring for at least one LBW infant, who was born 24-28 weeks premature, and had been discharged from the Neonatal Intensive Care Unit (NICU) for at least one month. On average, the infants had stayed in the NICU for 59 days.

All families were interviewed either in their homes $(n=15)$ or in the hospital where their infant was born $(n=3)$ as individuals $(\mathrm{n}=16)$ or in pairs $(\mathrm{n}=2)$. Interviews were conducted in English $(n=16)$ or Spanish $(n=2)$, according to the participant's comfort level. During the interview, our primary interest was to understand how parents kept track of the development and health of their preterm infants. In particular, we asked about the types of information parents recorded, the barriers to and tools used for data capture, and how parents made sense of their records. We also asked about parents' current practices for caring for their infants, their strategies for preparing for their discharge from the hospital, the quality of communication with their infants' healthcare providers, and their strategies for getting help or finding information about how to care for their infant. Interviews lasted 1 hour and were audio-recorded, transcribed, and translated when applicable.

To supplement our family interviews, we also interviewed 11 healthcare professionals either in groups (one group of seven and one pair) or as individuals $(n=6)$. These professionals included nurses, pediatricians, developmental psychologists, and social workers who all specialize in caring for preterm infants. Seven worked at a high-risk infant followup (HRIF) program affiliated with a 238-bed regional hospital that houses a 54-bed Level III NICU. The remaining professionals worked at a 422-bed academic medical center that houses a 45-bed Level III NICU. The HRIF program is responsible for state-mandated monitoring of most preterm infants in the surrounding geographic areas. These include any infants admitted to a hospital's NICU, as well as those who are considered high risk, either due to an illness or because they were born prematurely, but were not admitted to the NICU.

When interviewing the clinicians, we focused on their perceptions of parental concerns, how data collected by parents impacted clinical workflow, and any other concerns they had about caring for preterm infants and their families. Group interviews lasted several hours; individual interviews lasted one hour. Interviews were recorded and transcribed when permitted $(\mathrm{n}=8)$. In all cases, detailed notes were taken for analysis.

We coded all interview transcripts and field notes using an emergent open coding scheme to identify recurring themes related to how caregivers manage and monitor the health of their preterm infants. Additionally, we used themes from the literature (e.g., parental stress, inadequate feelings of selfefficacy) to confirm recognized challenges in our population. These themes developed iteratively over several months, with the entire research team meeting regularly to discuss the data.

\section{B. Phase 2: Participatory Design with Professionals}

Building on the findings from the first phase of this work, during August 2010 to August 2011, we used a participatory design process to develop Estrellita. Specifically, we invited four healthcare professionals to join our design team. All four professionals were recruited from the same HRIF program used in our first round of interviews. Three of the professionals had participated in the first phase of our design process, including two case managers and a nurse who manages the HRIF program. The fourth design team member is a psychologist who focuses on pediatric mental health and development.

We conducted ten participatory design sessions of approximately one to two hours each. During these sessions, we conducted several participatory design exercises, including ideation, card-sorting exercises, and low- and high-fidelity prototyping. Design sessions were recorded and transcribed when possible $(n=4)$. In all cases, detailed notes were taken for analysis. Between design sessions, conference calls occurred every two weeks among the entire design team, including both the professionals and the researchers, which enabled continued communication and feedback regarding the design of Estrellita. 
These participatory design sessions did not include parents. The significant time commitment for the design sessions is prohibitive to parents with preterm infants at home who are dealing with caregiving responsibilities that severely limit their availability. Also, all the clinicians in our design sessions are domain experts who have several years of experience with families caring for preterm infants. Thus, they bring insight into the challenges faced by both medical staff and families.

\section{Pediatric CAPture AND ACCess Challenges}

In this section, we describe our findings from the interviews conducted in phase one. To better illustrate the different needs of parents and clinicians, we discuss their concerns separately.

\section{A. Challenges for Parents Capturing Infant Health Data}

The transition from the NICU to home can be stressful for parents, because the level of care being asked of them is beyond that of the usual parental role [19]. At the same time, this first year is a critical time for growth and development for the infants. Yet, past work has shown that an infant's transition from the NICU to the home is poorly understood [20]. In our interviews, we asked parents to discuss their caregiving experiences, paying particular attention to their tracking of health and developmental data.

All of the parents in our study confirmed that the transition to in-home care was challenging. Parents also agreed that these challenges had not diminished over time, despite our interviews being an average of 8 months after NICU discharge. Although there are many caregiver stressors (e.g., worrying about the infant's health, caring for a sick child and other children simultaneously, etc.), in this section, we describe the particular challenges to data capture that made it difficult for parents to track and record their infant's health information.

1) Managing Infant Appointments Can Be Overwhelming: Nearly two-thirds of preterm and LBW infants continue to have medical and/or developmental concerns beyond the NICU [21]. As a result, many NICU graduates have significantly more clinic visits than NBW infants [20]. Managing these appointments can be overwhelming for parents, particularly when they use a variety of different doctors and service organizations. While these services are sometimes minimally coordinated in a referral system, the burden is still on parents to schedule and attend appointments.

\begin{abstract}
"When we wake up, I get [my baby] ready and check to see if I have appointments that day. I go to the doctor almost everyday. If it's not the lungs, then I have to go to for a shot...or I have to go to the pediatrician or the eye doctor, I mean, every day of the week [the baby and I] see a doctor." [P15, $\mathrm{P}=$ parent $]$

"I go [to the doctor's] for the ARSB injection, then a normal check up, then the lungs and heart specialist, and the one for the milk...[the baby] sees more than 5 doctors in one month." [P16]
\end{abstract}

It is not just the quantity of appointments that can be difficult for parents to track. They must also serve as the information conduit between physicians by communicating diagnoses and recommendations from one clinician to another. Most parents are not medical experts. Thus, relying on them to relay health information can lead to instances in which important details are forgotten, misunderstood, or miscommunicated. Without an accurate health record, it is much harder for clinicians to provide early interventions for potential health problems in the infant.

2) Uncertainties Regarding Infant Care: As parents transition from the NICU to home, they may feel overwhelmed with the responsibility of caring for their newborns, administering numerous medications, and observing milestones for potential symptoms of other health complications. Many parents feel unprepared for their infant's discharge [22], an issue that was echoed in our interviews, in which parents were worried both about immediate care after discharge and over long term development, particularly when compared to other infants who were born full-term.

\section{'...if [the baby] has a problem...I worry and then I remember I should not compare him to normal kids but with premature babies..." [P15] \\ "I have to educate [parents]... 'cause that can be a source of stress for families if their child is nine months old and they're not doing the things that other nine month olds do, but they're really like, you know, five or six months - that's a big difference." [C21, C=clinician, clinical social worker]}

Beyond concerns that arise from comparing with healthy full-term infants, parents also expressed alarm over similar issues to those of healthy infants. For example, parents may not understand what "normal" bowel movements are like for infants. These kinds of uncertainties regarding infant care can lead parents to not track the necessary health data:

"[The doctor] asked me how [the baby's] bowel movements are going... and I'm like 'I don't even know'...that's one thing I don't record. ', [P13]

At the same time, other parents may become unnecessarily alarmed. For example, one pediatrician described getting so many calls and visits about diaper concerns that he carries sample pictures of "normal poops" on his phone and emails them or shows them to concerned parents.

3) Inconsistent, Short-Term Data Capture: Parents commonly reported that, in response to clinician requests, they track data only immediately prior to the infant's appointments:

"If I know I'm going to the doctor and I know they're going to ask me a question, then I start keeping track... [but] it's not like, you know, a nice data form... it's like whatever I have around...like, a receipt with a pencil basically. [After the appointment] I just throw it away: 'Okay, that's done." ' [P11]

Outside of appointments, data capture can also be triggered by specific events. For example, parents were motivated to capture data when they thought there might be a problem.

“...I actually, like, kept a little, you know, log...Because his poop, it was worrying me. I was like, 'He's not going. He's not having a bowel movement. '... ” [P11] 
Event-triggered data capture, as opposed to a more continuous model, can result in missing information from before the event emerges. For example, it may have been more meaningful for P11 to collect data prior to her observation of her baby's lack of bowel movements to determine what factors may have led to the change in her infant's bowel movements.

4) Fixation on Data Capturing: When parents we interviewed were diligent about capturing infant data, they described the act of recording as causing substantial additional burdens. For example, one parent described being so concerned with tracking her infant's health data that she lost track of important events in her baby's life.

"I take down feedings, every cc...that was such an added stress...that alone is so hard to do...I can tell you every date of surgery the first year of my son's life, [but]

I don't know the month he got his first tooth...so there were specific things that I just missed...the things that you would think you would know - no clue." [P20]

An approach like that used in the BabySteps application [13] could enable parents to record both health data and more personally interesting data. However, this solution does not address the concerns, shared by parents and clinicians, that tracking such data can raise unnecessary concerns. For example, weight gain is a major component of an infant's clinical visits and, in some cases, parents may be asked to bring their infants in for weight checks between appointments. However, parents are often so concerned about weight that they monitor it closely even between their appointments:

“...lots of times I've heard of moms who take their kids to the grocery store and put them on the little scale just to see if they're gaining weight." [C19, nurse and HRIF coordinator]

This approach can be problematic, because day to day variations may alarm parents unnecessarily. These results indicate that a balance must be found between asking parents to record data so frequently that they become alarmed by false positives and allowing parents to record data so infrequently that they produce false negatives.

\section{B. Challenges for Clinicians Accesing Infant Health Data}

Beyond the challenges that families face in data capture, pervasive health tools that support this population must also consider the concerns of clinicians. In particular, these individuals must access the data captured by the parents, understand it, and act upon it. In this section, we describe the results of our interviews with clinicians, focusing on the struggle they reported in helping parents understand how they should track their infant's health and what data to collect.

1) Parent-Infant Dyad: Parental health is strongly linked to infant health. For example, maternal post-partum depression has been shown to adversely affect infant development [23] and may be as high as $40 \%$ among mothers of preterm infants [24]. Although not clinically trained to care for these mothers, the professionals we interviewed all described being concerned about maternal mental health. For example:

"We want to make sure we're taking care of the whole family...we make sure that [the] mom is not depressed, that there is not any post-partum happening, that we're not seeing any red flags [for the mom]." [C20, HRIF liason between families and medical staff]

Aside from post-partum depression, stress has also been shown to adversely affect infant development. High levels of parental stress have been correlated with maladaptive parentinfant interactions $[25,26]$ and lower cognitive resilience in infants [27]. Consequently, clinicians ask about parental stress and emphasize the importance of monitoring their own health.

"I ask the family if...there are a lot of stressors, I will ask them...what are they doing to take care of themselves?... Because it's really hard a lot of times when you have a very involved child it's all about them and you forget about yourself." [C21]

Despite recognizing these links, very few US professionals care for families as a whole. Rather, mothers receive their primary care from a obstetrician/gynecologist or a general physician, while preterm infants receive care from pediatricians and specialists. As a result, an important part of an infant's wellbeing can be missing in their health records.

2) Parents as Inter-Clinician Intermediary, Limited Data Aggregation and Trending: The lack of shared data among healthcare providers about specific patients results in parents acting as the primary communicators of an infant's medical history. This includes the infant's overall health, any healthrelated incidents prior to the appointment, and treatment regimens prescribed by other physicians. For example, at the HRIF six-month assessment appointment, clinicians try to ascertain the infant's medical history since discharge:

"When [parents] come in you ask them who is [the baby's] doctor? Do[es] [the baby] have any allergies? When did he see the doctor last? Why did he see the doctor? Did he get his immunizations? And this is all information that is entered in our database... " [C19]

Professionals, particularly clinical health providers, also have limited time to provide care. During short appointments (typically less than 15 minutes), they must assess the physical health of the infant, answer parent questions, and go over any information that has been shared with them, either through health records systems or by parents bringing data to the appointment. The clinicians we interviewed observed two general types of data reporting behaviors adopted by parents. On one hand, there were parents who were so stressed that it was difficult for them to accurately remember the necessary information from all of their previous appointments. On the other hand, there were parents who presented the clinicians with so much data that it was too cumbersome to examine.

"At an appointment...[a] mom came in with, like, a print out or something of, like, every single data point...it's going to take too much time and energy to make sense out of [the data]." [C23, psychologist specializing in infant and toddler development]

This situation can be mitigated if professionals are able to view data trends rather than raw data. For example, one clinician commented that tracking infants' weight is an 
important health indicator, but the exact weight is not nearly as informative as whether there are consistent weight gains.

"For [pediatricans] ...they just watch the curve of [the baby's] weight. They don't care what happen in between... as long as the curve's going right... " [C19]

These results indicate that visualizing historical data to enable professionals to spot trends efficiently may encourage them to view and act on patient-generated data.

3) The Importance of Non-Health Data: Preterm infants represent complex, rapidly developing health cases. As such, holistic care includes understanding and monitoring a variety of non-health related data. Because appointment attendance is particularly problematic for this population, clinicians monitoring an infant's overall wellbeing (e.g., the HRIF staff, a primary care pediatrician, and so on) will also be interested to see if families have missed any of their other appointments.

Beyond this kind of pragmatic information, professionals also expressed the need for other kinds of non-health data. For example, bonding with preterm infants can be challenging for parents who may be concerned about the infant's medical fragility and so this must be monitored and supported.

"The families have an impaired relationship because...they're struggling with having a child who is ultra-fragile...If the parents parent that child in such a way that they're completely fragile...then that child is going to develop a lot of issues around that." [C22, director of a regional HRIF program]

Once a close bond is established, professionals also reported using parental knowledge of temperament to diagnose and treat their patients. Monitoring an infant's moods can help identify particular challenges. For example, an infant who becomes upset when laid on his back may suffer from acid reflux. Such monitoring can also enable higher quality care. For example, HRIF specialists need information about schedules to help make referral appointments:

\begin{abstract}
"We don't know his likes and his dislikes and his discomforts. Only parents can do that...[For example,] if the child is really tired in the morning, it doesn't make any sense for us to make a recommendation for PT [physical therapy] in the morning. " [C20]
\end{abstract}

\section{Estrellita: A Mobile Health Informatics ToOL}

Drawing from our interview findings, we designed Estrellita (Figure 1) to consider the capture and access needs for both families and clinicians. In particular, we draw on our co-design activities with the clinicians to illustrate how we resolved tensions round designing for diverse stakeholders.

\section{A. Enable Parent-Clinician Communication \& Coordination}

Poor appointment attendance rates are correlated with higher risks of developmental complications in preterm infants [28]. Although maintaining a current calendar is an important first step for families dealing with a myriad of clinical appointments, knowing when and where these obligations are is not enough. Thus, systems designed to support families of preterm infants should include reminders and opportunities for reflection to encourage parents to attend appointments, get support when appointments are missed, and communicate with clinicians during the appointments.

Estrellita reminds parents two days prior to scheduled appointments. During this reminder, parents are encouraged to review any notes they have attached to the appointment. Parents also answer questions about how likely they are to attend and if there are any specific challenges prohibiting their attendance. This information is then shared with HRIF personnel who support rescheduling or provisioning of resources (e.g., child care, transportation). Additionally, an appointment likely to be missed generates a daily reminder for the next three days to reschedule the appointment.

After an appointment, parents should reflect on what was told to them and schedule follow-up appointments, if needed. Thus, Estrellita asks parents three hours after a scheduled appointment to report on who attended the appointment (and, if it was not attended, the system proactively reminds them to reschedule), how helpful they found the appointment, and how much they remember and understood of what the doctor said. These responses are forwarded to HRIF clinicians who intervene if needed by explaining information the parent received or helping to schedule a follow-up appointment.

Beyond supporting parents in medically caring for their infants, clinicians can also provide social support through guidance, feedback and positive social interaction [5]. Estrellita enables the exchange of text messages with clinicians through a custom inbox. Parents can use Estrellita to take photographs of potential medical issues (e.g., problematic diaper outputs), so that clinicians can be better informed of potential problems when replying to parents' concerns. Additionally, a "virtual coach" offers system-generated support for parents by posting daily messages of encouragement and advice.

\section{B. Support Many Data Types, Require Only a Few}

Tracking too much information can be problematic for parents and professionals. However, each infant is unique and may require particular information to be collected. Thus, pervasive health solutions should support a variety of data and information types but only require that a limited set be tracked. Estrellita supports four classes of health data: clinically relevant pediatric health data, parental indicators of wellbeing, pragmatic care information, and custom information.

Estrellita enables tracking of two clinically relevant health indicators: weight and diapers (Figure 1b). As previously mentioned, low birth weight means that weight gain is a continual concern for preterm infants. Additionally, preterm infants are at risk for gastrointestinal infections that can present as problematic digestive outputs (e.g., bloody or irregular output) $[29,30]$; thus, there is a need to track diaper output.

Estrellita enables monitoring of three indicators of parental mental health and wellbeing: post-partum depression (using the Edinburgh Postnatal Depression Scale [31]), stress levels (using the Perceived Stress Scale [32]), and a subjective mood indicator (using Intel's Mood Map [33]). Through regular surveys using validated instruments, Estrellita automatically assesses parental risk for post-partum depression and provides resources and forwards the information to a clinician for a referral if a problematic score is returned. Additionally, 

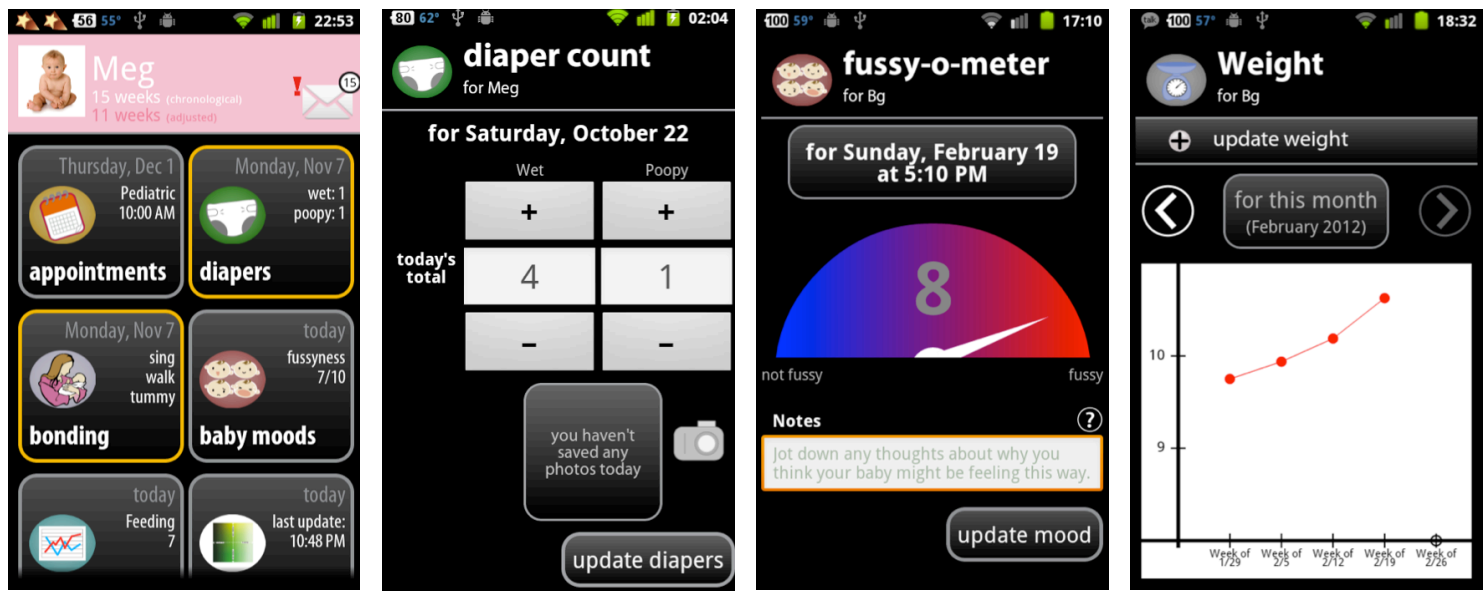

Figure 1. Estrellita screenshots, from left to right: a) the main screen

showing a summary of all of the data captured by the parent; b) the form used to record infant diapers; c) the fussyo-meter used to record the infant's irritability; d) a visualization showing the infant's weight data for the past month.

Estrellita encourages parents to review their own mental health through a visualization of their past mood entries.

Estrellita also records non-health data that can be useful for assessing the infant's overall health. For example, Estrellita allows parents to track how "fussy" an infant is (Fig 1c), which allows parents and professionals to look for patterns related to time of day, caregiver mood, or other factors impacting the infant's temperament. Although this symptom is subjective, much like parental mood, it can serve as an important precursor to something more problematic. To encourage parents to engage in activities that help them bond with their infant (e.g., singing, talking, reading) and to attend appointments on time, Estrellita supports tracking of both of these elements and reflects back to the parents when they have completed them.

Finally, although our goal was to minimize the amount of data to be tracked and therefore reduce the burden on caregivers, some infants require tracking of other types of data, such as administration of medication. Thus, Estrellita provides the option for parents to create custom charts and graphs to support tracking of either textual data (e.g., the types of medicine an infant takes) or numerical data (e.g., the number of ounces of liquid medicine that an infant has been fed).

\section{Encourage Consistent Data Capturing Routines}

Because parents can be inconsistent in tracking data and often record information only when they sense that there might be a problem with their infant's health or just before an appointment, the window of relevant health data may be much wider than the period of actual recording. Thus, Estrellita is designed to encourage parents to track health data regularly and consistently. For health indicators like diaper counts and bonding activities, Estrellita provides daily reminders for parents to input data. Reminders are provided explicitly (using tactile and audio alerts every evening, Figure 2b), ambiently (using a dynamically updated widget placed on the phone's home screen, Figure 2c), and subtly (using simple visual cues on the application's launching screen, Figure 2a).

Just as Estrellita provides cues to encourage routine data capture, it also aims to reduce problematic over-recording and fixation on health data. Thus, for data elements likely to raise false alarms, such as infant weight and some measures of parental mental health, Estrellita only allows parents to enter data weekly or monthly. This rate of data capture is sufficient to inform clinicians of important trends over time (i.e., weight gains/loss), without unnecessarily overwhelming the parent with natural day-to-day fluctuations.

\section{Provide Opportunities for Reflection and Communication}

To facilitate communication between parents and clinicians, Estrellita provides glanceable charts that show a historical view of health data (Figure 1d). Individual data points on the graph are clickable to allow sharing of any notes parents may have attached to a recorded data point. All health data are also stored
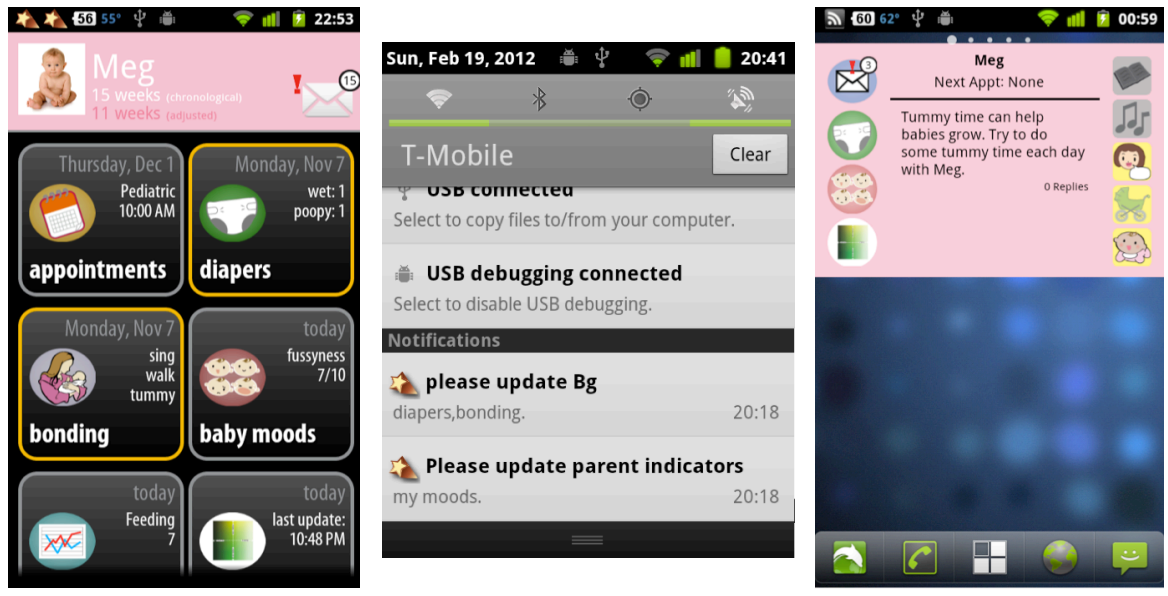

Figure 2. Screenshots of the different reminders embedded in Estrellita, from left to right: a) on the main screen, tiles that have an orange border (vs. a gray border) indicate that the parent has not recorded that particular data recently; $b$ ) nightly reminders show up in the phone's system tray which triggers a tactile and an audio alert on the phone; c) the Estrellita widget is placed on the phone's home screen and provides a quick glance of upcoming appointments, the latest message from the clinician or the virtual coach, and the visual cues of whether the parent has completed a particular bonding activity for that day (cues that have yellow backgrounds indicate that the activity has been completed; icons with a gray background mean that the activity has not yet been completed) 
in an online personal health record that can be shared with clinicians or joined with clinical health records that support such integration.

Reflection has been shown to be an integral part of data capturing. However, pure self-reflection (i.e., reflecting without the aid of a tool) is often flawed because people have limited memory and may not have the time to consistently record the relevant health data [34]. Reflecting with the aid of a pervasive health tool can alleviate many of these difficulties. Additionally, by viewing the visualizations, parents can be more proactive in how they manage their infant's health because trends and patterns will appear more easily. Thus, for each type of information, a visualization is displayed prior to data entry in order to encourage parents to reflect on their previously recorded health data.

\section{DESIGN LESSONS FoR HEALTH INFORMATICS TOOLS}

In this paper, we described our two-part design process including both fieldwork and participatory design. The findings from our design process resulted in actionable design guidelines that, in turn, formed the basis for Estrellita, our mobile health informatics tool for preterm infants and their caregivers. In this section, we describe three insights we learned from our process and discuss how they can apply to other pervasive health solutions.

\section{A. Empowering Parents Through Reflection and Awareness}

Empowering parents to care for their preterm infants can lead to improved health outcomes. In particular, interventions targeting LBW infants produced significantly better results when parents were actively involved and interested in the intervention activities [35]. Empowering parents can enhance confidence and satisfaction with the baby, further strengthening family bonds and improving their ability to rise to the challenge of caring for a preterm infant [7].

In Estrellita, we facilitate caregiver empowerment in two ways. First, Estrellita provides several feedback mechanisms (e.g., charts, encouragement from virtual coach, messages from clinicians, etc.) that can increase parents' awareness of their child's health status and potentially bolster parental feelings of self-efficacy as a caregiver. Second, Estrellita streamlines its data capturing to focus on a few, but important, data types that help to give a holistic view of the infant's health. Estrellita guides the data entry process using simple forms and regular reminders in an effort to educate parents, equipping them to better care for their infants [36].

Many personal informatics tools incorporate principles of self-reflection into their designs [34], which can lead to feelings of patient empowerment. However, in pediatric care as well as in eldercare - a caregiver, not the patient, is the predominant user of the capture and access tool. In these cases, special care must be taken to ensure that the caregiver continues to be motivated and supported in their care routines.

\section{B. Tradeoffs in Levels of Automation for Data Entry}

Determining the appropriate amount of automation for data collection in a pervasive health tool can be a substantial challenge. Explicit data entry can help reinforce the collection process and motivate the caregiver to transition to a reflection phase of discovery and/or analysis for the data they are collecting [34]. On the other hand, manual data collection can overburden families and lead to errors in data capture (both in the form of missing data and erroneously recorded data).

When automated data collection (e.g., through sensors) is used, tools must provide explicit opportunities for feedback and reflection. Without intentional reflection, automated capture and access applications can lead to an over-reliance on automatic analysis and lessen empowerment opportunities for the caregiver. Thus, finding a balance between system- and user-generated data capturing is an important design challenge for pervasive health systems.

\section{Collaborative Prescriptions for Data Capture}

We incorporated feedback from multiple stakeholders throughout our design process. This inclusion of a wide variety of relevant stakeholders required us to balance the sometimesconflicting needs of clinicians and caregivers. In Estrellita, one of the ways we balance these design tensions is to more heavily emphasize the importance of collecting clinical health data (e.g., weight and parental wellbeing), while also providing (but not requiring) the use of custom charts for more individualized data collection needs.

Although pervasive health researchers and designers have long argued for this type of collaborative inquiry across multiple stakeholder groups when conducting research, incorporation of all these perspectives is less common in clinical practice. Thus, not only should future applications for these and other chronic health populations emerge from a multi-perspective design process, but they should also enable customization during use. This customization can support further adaptation to the needs of all relevant stakeholders. Clinicians, caregivers, and patients should be able to collaboratively generate diagnosis-specific "prescriptions" for data capture. With such tools, caregivers and patients are better equipped to capture and present their data in a format that is consumable by clinicians and fits into current work practices.

\section{CONCLUSION AND FUtURE WORK}

HRIF programs can mitigate functional limitations for preterm infants and reduce the risk of delays in cognitive, motor, and other skills. However, these interventions can be cost-prohibitive and require substantial resources. In this paper, we presented the results of a two-part design process with the goal of developing a system that could reduce costs associated with these programs while improving data collection, health outcomes, and caregiver feelings of self-efficacy.

Our results reveal diverse challenges facing parents and clinicians attempting to document, understand, and share infant health data that must be balanced in any pervasive health solution. In particular, parents described struggling with when to collect data, while clinicians reported being more concerned about what and how parents track infant health. The design implications presented here are based on these findings and were incorporated into the design of Estrellita, a mobile health informatics tool to support caregivers of preterm infants. Although some design challenges and open research questions remain, our design of Estrellita demonstrates one potential pervasive health solution to support the diverse caregiving 
needs of parents and professionals, Additionally, the design guidelines used for Estrellita are applicable to other pediatric pervasive health applications. Understanding the impact of these approaches is an open question for a long-term deployment study. In particular, we plan to probe issues around parent engagement and the potential for disparate needs between parents and clinicians.

\section{ACKOWLEDGEMENTS}

This work is supported by the Robert Wood Johnson Foundation Project HealthDesign, NSF grant 0846063, and Google. We thank Monica Tentori and Leslie Liu for their efforts in early stages of this research. We also thank the participants in our study for their time. This work is approved by the UC Irvine IRB under protocol HS\# 2010-7933.

\section{REFERENCES}

[1] J.A. Martin, B.E. Hamilton, S.J. Ventura, M.J.K. Osterman, S. Kirmeyer, T.J. Mathews et al., "Births: Final Data for 2009 " Nat'l Vital Statistics Reports, vol. 60, no. 1, pp. 1-104, 2011.

[2] M. Hack, H.G. Taylor, D. Drotar, M. Schluchter, L. Cartar, L. Andreias et al., "Chronic Conditions, Functional Limitations, and Special Health Care Needs of School-aged Children Born With Extremely Low-BirthWeight in the 1990s," JAMA, vol. 294, no. 3, pp. 318-325, 2005.

[3] M.A. Slater, M. Naqvi, L. Andrew, and K. Haynes, "Neurodevelopment of monitored versus nonmonitored very low birth weight infants: the importance of family influences," J Dev Behav Pediatr, vol. 8, no. 5, pp. 278-285, 1987.

[4] M.K. Campbell, E. Halinda, M.J. Carlyle, A.M. Fox, L.A. Turner, and G.W. Chance, "Factors Predictive of Follow-up Clinic Attendance and Developmental Outcome in a Regional Cohort of Very Low Birth Weight Infants," Am J Epidemiol, vol. 138, no. 9, pp. 704-713, 1993.

[5] L.S. Liu, S.H. Hirano, M. Tentori, K.G. Cheng, S. George, S.Y. Park et al., "Improving communication and social support for caregivers of high-risk infants through mobile technologies," in CSCW '11, 2011, pp. 475-484.

[6] A.P. Winrow, "Growing up: The future for neonatal unit graduates," Infant, vol. 8, pp. 234-237, 2007.

[7] S. Cockcroft, "How can family centred care be improved to meet the needs of parents with a premature baby in neonatal intensive care?," $J$ Neonatal Nurs, vol. in press, 2011.

[8] R.N. Shiffman, S.A. Spooner, K. Kwiatkowski, and P.F. Brennan, "Information technology for children's health and health care: report on the Information Technology in Children's Health Care Expert Meeting," JAMIA, vol. 8, pp. 546-551, 2001.

[9] J.E. Gray, C. Safran, R.B. Davis, G. Pompilio-Weitzner, J.E. Stewart, L. Zaccagnini et al., "Baby CareLink: Using the Internet and Telemedicine to Improve Care for High-Risk Infants," Pediatrics, vol. 106, no. 6, pp. 1318-1324, 2000

[10] Y.S. Lee, C. Garfield, N. Massey, S. Chaysinh, and S. Hassan, "NICU2-HOME: supporting the transition to home from the neonatal intensive care unit using a mobile application," in CHI '11 Extended Abstracts, 2011, pp. 2257-2262.

[11] H.Y. Jeong, S.Y. Park, and J. Zimmerman, "Opportunities to support parents in managing their children's health," in CHI '08 Extended Abstracts, 2008, pp. 3225-3230.

[12] G.R. Hayes, J.A. Kientz, K.N. Truong, D.R. White, G.D. Abowd, and T. Pering, "Designing Capture Applications to Support the Education of Children with Autism," in Ubicomp '04, 2004, pp. 161-178.

[13] J.A. Kientz, R.I. Arriaga, M. Chetty, G.R. Hayes, J. Richardson, S.N. Patel et al., "Grow and know: understanding record-keeping needs for tracking the development of young children," in CHI '07, 2007, pp. 1351-1360.

[14] G.D. Abowd, and E.D. Mynatt, "Charting past, present, and future research in ubiquitous computing," ACM Trans. Comput.-Hum. Interact., vol. 7, no. 1, pp. 29-59, 2000.

[15] Trixie Tracker. "Baby Tracker for iPhone, Android and Web," February 16, 2012; http://www.trixietracker.com/.
[16] J. Blomberg, J. Giacomi, A. Mosher, and P. Swenton-Wall, "Ethnographic Field Methods and Their Relation to Design," Participatory Design: Principles and Practices, D.a.N. Schuter, A., Editors, ed., pp. 123-155: Lawrence Erlbaum Assoc, 1993.

[17] J. Hughes, King, V., Rodden, T., and Andersen, H. , "The Role of Ethnography in Interactive Systems Design," Interactions, vol. 2, no. 2, pp. 56-65, 1995.

[18] M.J. Muller, and S. Kuhn, "Participatory design," Comm of ACM, vol. 36, no. 6 , pp. 24-28, 1993.

[19] Committee on Fetus and Newborn, "Hospital Discharge of the HighRisk Neonate--Proposed Guidelines," Pediatrics, vol. 102, pp. 411-417, 1998.

[20] K.C. Wade, S.A. Lorch, S. Bakewell-Sachs, B. Medoff-Cooper, J.H. Silber, and G.J. Escobar, "Pediatric care for preterm infants after NICU discharge: high number of office visits and prescription medications," $J$ Perinatology, vol. 28, pp. 696-701, 2008.

[21] V. Fornari, M. Sherman, and E. Lipper, "Parental needs in a neonatal intensive care follow-up clinic," Pediatric Research, vol. 18, 1984.

[22] N. Sneath, "Discharge teaching in the NICU: are parents prepared? An integrative review of parents' perceptions.," Neonatal Netw., vol. 28, no. 4, pp. 237-246, 2009.

[23] S.L. Grace, A. Evindar, and D.E. Stewart, "The effect of postpartum depression on child cognitive development and behavior: A review and critical analysis of the literature," Archives of Women's Mental Health, vol. 6, no. 4, pp. 263-274, 2003.

[24] S. Vigod, L. Villegas, C.L. Dennis, and L. Ross, "Prevalence and risk factors for postpartum depression among women with preterm and lowbirth-weight infants: a systematic review," BJOG, vol. 117, no. 5, 2010.

[25] C. Muller-Nix, M. Forcada-Guex, B. Pierrehumbert, L. Jaunin, A Borghini, and F. Ansermet, "Prematurity, maternal stress and motherchild interactions," Early Hum Devel, vol. 79, no. 2, pp. 145-158, 2004.

[26] R. Pianta, and B. Egeland, "Life stress and parenting outcomes in a disadvantaged sample: Results of the mother-child interaction project," $J$ Clinical Child Adol Psych, vol. 19, no. 4, pp. 329-336, 1990.

[27] M. Tu, R. Grunau, J. Petrie-Thomas, D. Haley, J. Weinberg, and M. Whitfield, "Maternal stress and behavior modulate relationships between neonatal stress, attention, and basal cortisol at 8 months in preterm infants," Devel Psychobiology, vol. 49, no. 2, pp. 150-164, 2007.

[28] M. Ballantyne, "Maternal-infant Predictors of Attendance at Neonatal Follow-up Programs," Ph.D. Dissertation, Graduate Department of Institute of Medical Science, University of Toronto, Toronto, Canada, 2010

[29] S. Blackburn, "Problems of Preterm Infants After Discharge," $J$ Obstetric, Gynecologic, \& Neonatal Nurs, vol. 24, no. 1, pp. 43-49, 1995.

[30] J. Neu, "Gastrointestinal development and meeting the nutritional needs of premature infants," Am J Clin Nutr, vol. 85, no. 2, pp. 629S-634S, 2007.

[31] J.L. Cox, J.M. Holden, and R. Sagovsky, "Detection of postnatal depression. Development of the 10-item Edinburgh Postnatal Depression Scale," BJP, vol. 150, no. 6, pp. 782-786, 1987.

[32] S. Cohen, T. Kamarck, and R. Mermelstein, "A global measure of perceived stress," J Health Social Beh, vol. 24, no. 4, pp. 385-396, 1983.

[33] M.E. Morris, Q. Kathawala, T.K. Leen, E.E. Gorenstein, F. Guilak, M. Labhard et al., "Mobile Therapy: Case Study Evaluations of a Cell Phone Application for Emotional Self-Awareness," JMIR, vol. 12, no. 2, pp. e10, 2010.

[34] I. Li, A. Dey, and J. Forlizzi, "A stage-based model of personal informatics systems," in CHI '10, 2010, pp. 557-566.

[35] F.R. Liaw, S.J. Meisels, and J. Brooks-Gunn, "The effects of experience of early intervention on low birth weight, premature children: The Infant Health and Development Program," Early Child Research Qtrly, vol. 10, no. 4, pp. 405-431, 1995.

[36] B. Lindberg, and K. Ohrling, "Experiences of having a prematurely born infant from the perspective of mothers in northern Sweden," Int $J$ Circumpolar Health, vol. 67, no. 5, pp. 461-471, 2008. 